\title{
Autosomal recessive congenital cerebellar ataxia due to GRID2 deficiency
}

INSERM

\section{Source}

INSERM. (1999). Orphanet: an online rare disease and orphan drug data base. Autosomal recessive congenital cerebellar ataxia due to GRID2 deficiency. ORPHA:363432

Autosomal recessive congenital cerebellar ataxia due to GRID2 deficiency is a rare, genetic, slowly progressive neurodeg enerative disease resulting from GRID2 deficiency characterized by motor, speech and cognitive delay, hypotonia, truncal and appendicular ataxia, and eye movement abnormalities (tonic upgaze, nystagmus, oculomotor apraxia). Intention tremor may also be associated. Brain imaging reveals progressive cerebellar atrophy with cerebellar flocculus particularly affected. 